\title{
High expression of MAPK-14 promoting the death of chondrocytes is an important signal of osteoarthritis process
}

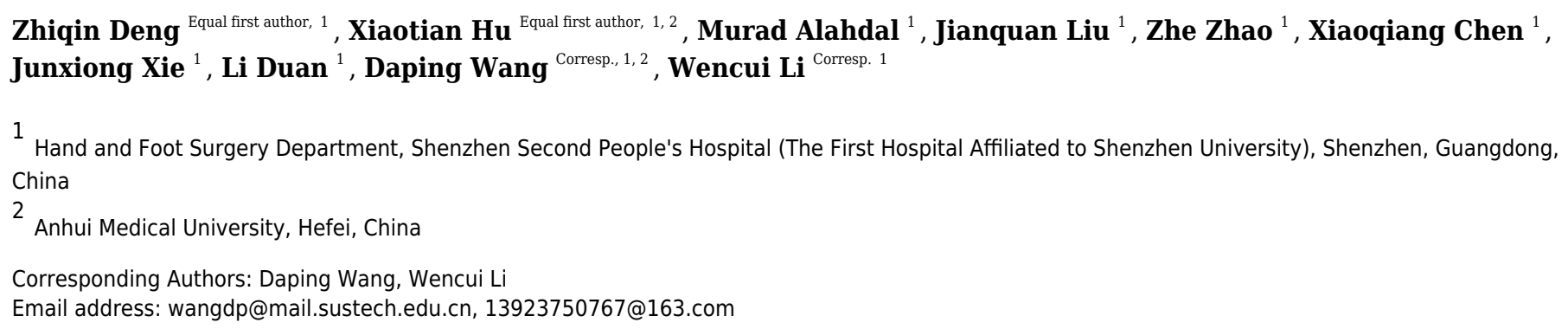

Background Osteoarthritis (OA) is one of the most common degenerative diseases worldwide. Many researchers are studying the pathogenesis of OA, however, it is still unclear. Methods Screening and validation of OA relevant hub genes are an important part of exploring their potential molecular mechanism. Therefore, this study aims to explore and verify the mechanisms of hub genes in the OA by bioinformatics, qPCR, fluorescence and propidium iodide staining. Results Microarray datasets GSE43923, GSE55457 and GSE12021 were collected in the Gene Expression Omnibus (GEO), including 45 samples, which divided into 23 osteoarthritis knee joint samples and 22 samples of normal knee joint. Thereafter, 265 differentiallyexpressedgenes (DEGs) were identified in all, which divided into 199 upregulated genes and 66 downregulated genes. The hub genes MAPK-14, PTPRC, PTPN12 were upregulated, while B9D1 was downregulated. In order to further confirm the expression of screening differential genes in human chondrocytes, the human chondrocytes were extracted from a joint replacement surgery and stained with toluidine blue for identification. Compared with normal chondrocytes, OA chondrocytes had high expression of COL I protein and low expression of COL II protein. The expression levels of MAPK-14, PTPRC and PTPN12 in OA chondrocytes were significantly higher than the expression levels of B9D1 in normal chondrocytes. Moreover, the inflammatory necrosis of OA chondrocytes was increased compared with the normal chondrocytes by propidiumiodide staining. Conclusions The high expression of MAPK-14 works as a promoter of chondrocytes death and an important signal of the osteoarthritis process. 
1

2 High expression of MAPK-14 promoting the death of chondrocytes is an important signal

3 of osteoarthritis process

4

5 Zhiqing, Deng ${ }^{1,}$ a , Xiaotian $\mathrm{Hu}^{1,2, \text { a, Murad Alahdal }}{ }^{1}$, Jianquan Liu ${ }^{1}$, Zhe Zhao ${ }^{1}$, Xiaoqiang

6 Chen ${ }^{1}$, Junxiong $\mathrm{Xie}^{1}$, Li Duan ${ }^{1}$, Daping Wang ${ }^{1,2, *}$, Wencui $\mathrm{Li}^{1,}{ }^{1}$

7

$8{ }^{1}$ Hand and Foot Surgery Department, Shenzhen Second People's Hospital (The First Hospital

9 Affiliated to Shenzhen University), Shenzhen, China.

${ }^{2}$ Anhui Medical University, Hefei, Anhui province, China.

11

12

Corresponding Author:

* Daping Wang ${ }^{1,2, *}$, Wencui $\mathrm{Li}^{\mathrm{b}}$, *

Daping Wang, Shenzhen Second People’s Hospital, 3002 Sungang West Road, Shenzhen 518000, Tel.: +86-0755-83791866, Fax: +86-0755-83366388,

E-mail: dapingwang1963@qq.com.

7 Wencui Li, Shenzhen Second People’s Hospital, 3002 Sungang West Road, Shenzhen 518000, Tel.: +86-0755-83366388, Fax:+86-0755-83366388, E-mail: 13923750767@163.com. ORCID ID: 0000-0003-2787-5360.

\section{a Zhiqin Deng, and Xiaotian Hu contributed equally to this work.}

Disclosure of potential conflicts of interest: There are no potential conflicts of interest.

\section{Abstract}

Background Osteoarthritis (OA) is one of the most common degenerative diseases worldwide.

Many researchers are studying the pathogenesis of OA, however, it is still unclear. 
26 Methods Screening and validation of OA relevant hub genes are an important part of exploring

27 their potential molecular mechanism. Therefore, this study aims to explore and verify the 28 mechanisms of hub genes in the OA by bioinformatics, qPCR, fluorescence and propidium 29 iodide staining.

30 Results Microarray datasets GSE43923, GSE55457 and GSE12021 were collected in the Gene 31 Expression Omnibus (GEO), including 45 samples, which divided into 23 osteoarthritis knee 32 joint samples and 22 samples of normal knee joint. Thereafter, 265 differentially expressed genes 33 (DEGs) were identified in all, which divided into 199 upregulated genes and 66 downregulated 34 genes. The hub genes MAPK-14, PTPRC, PTPN12 were upregulated, while B9D1 was 35 downregulated. In order to further confirm the expression of screening differential genes in 36 human chondrocytes, the human chondrocytes were extracted from a joint replacement surgery 37 and stained with toluidine blue for identification. Compared with normal chondrocytes, OA 38 chondrocytes had high expression of COL I protein and low expression of COL II protein. The 39 expression levels of MAPK-14, PTPRC and PTPN12 in OA chondrocytes were significantly 40 higher than the expression levels of B9D1 in normal chondrocytes. Moreover, the inflammatory 41 necrosis of OA chondrocytes was increased compared with the normal chondrocytes by 42 propidium iodide staining.

43 Conclusions The high expression of MAPK-14 works as a promoter of chondrocytes death and 44 an important signal of the osteoarthritis process.

46 Key words: Osteoarthritis; MAPK-14, chondrocytes, differentially expressed genes; protein47 protein interaction; Gene Ontology; 


\section{Introduction}

50 Osteoarthritis is the most serious chronic bone disease and can cause severe pain and even

51 disability in the elderly(Song et al. 2006). OA not only brings suffering to patients but also

52 aggravates their economic burden. Nearly half of the elderly patients are receiving treatment, and

53 most of them are still suffering or disabled(McKenzie \& Torkington 2010). About one-third of

54 elderly over 60 years old are supposed to be diagnosed with OA by 2030(Croft 2005). One of the

55 most important risks in OA development is the pathogenic changes of hub genes (Hochberg et al.

56 2013). Heritability of osteoarthritis reached approximately 50\% (Cibrian Uhalte et al. 2017).

57 However, the molecular mechanism genetic changes in OA remains unknown.

58 However, current researches showed some disadvantages such as using small amount of genetic samples and the lack of relevant experimental verification. Therefore, the expanding of the number of screening sample size on the basis of the predecessors, optimizing the relevant screening conditions and carrying out the relevant verification through cell lines experiments could contribute to further understanding and enhances further study of the internal mechanism regarding the occurrence and development of osteoarthritis. Recently, microarray technology and bioinformatics have made significant advances which contribute to understand differentially expressed genes (DEGs) in OA and enable us to do further studies. Here, we got 3 datasets from Gene Expression Omnibus (GEO) for analysis and obtain DEGs between OA knee joint samples and normal knee joint samples. Based on this, we haven't only analyzed Gene Ontology (GO), Kyoto Encyclopedia of Genes and Genomes (KEGG) pathway enrichment of DEGs, but also produced protein-protein interaction (PPI) network to help us unlock some of the unstudied parts of the molecular mechanisms of OA. The purpose of this research was to explore regarding underlying mechanisms of OA, so as to promote the further development of OA treatment. 


\section{Materials \& Methods}

\section{2.1. Microarray Data Information}

75 The Gene Expression Omnibus (GEO, http://www.ncbi.nlm.nih.gov/geo) is a genomic

76 database for all users, from which users can get all kinds of data free of charge(Barrett et al. 77 2013).The expression profiles of GSE43923, GSE55457 and GSE12021 were all searched and 78 got from the GEO. GSE43923 include 3 OA knee joint samples and 3 normal knee joint samples. 79 GSE12021 include 10 OA knee joint samples and 9 normal knee joint samples, and GSE55457 80 include 10 OA knee joint samples and 10 normal knee joint samples.

\subsection{Identification of DEGs}

83 GEO2R (http://www.ncbi.nlm.nih.gov/geo/geo2r), a free public network platform, was used to process the DEGs between OA knee joint samples and normal knee joint samples. Using GEO2R, we have integrated and analyzed multiple datasets for identifying DEGs. $|\log \mathrm{FC}|>1$ and P-value $<0.05$ were considered the cutoff criterion and have statistically significant.

\subsection{GO and KEGG enrichment analyses of DEGs}

DAVID(http://david.ncifcrf.gov), a free public online biological information database was used to provide biological function analysis for a large number of genes and can visualize the results(Huang et al. 2007). GO is a biological information analysis tool that can induce and analyze a large number of genes and visually export corresponding results, including BP, CC, and MF(Shannon et al. 2003). KEGG is a utility database resource for understanding advanced sequencing and other high-throughput experimental technologies from molecular level 
97 implemented by using the DAVID online database. $\mathrm{P}<0.05$ was considered statistically

98 significant.

99

\subsection{PPI network construction and module analysis.}

101

STRING(https://string-db.org/cgi/input.pl), a free public online search platform was used to

elucidate the relationship between interacting genes(Franceschini et al. 2013). DEGs previously screened in this research were imported into STRING to product the PPI network, and interaction with a confidence score $>0.4$ was considered significant. Cytoscape, a free and public source bioinformatics application that we imported the data into it. The built-in APP Molecular Complex Detection (MCODE) of Cytoscape is convenient to select the most significant modules in all DEGs and identify the most prominent gene in each module(Bader \& Hogue 2003).

\subsection{Isolation and culture of chondrocytes}

Cartilage specimens of the fracture patients' femoral head and the knee joint of patients with OA in Shenzhen second people's hospital were collected to isolate and cultivate chondrocytes.

112 The adherent cells were cultured in the chondrocyte medium, which contains the DMEMLG(Gibco), 10\%FBS(Gibco),1\% penicillin-streptomycin (Gibco), 1\%HEPES, 1\% vitamin solution, $1 \%$ proline solution and $1 \%$ non-essential amino acid NEAA. These adherent cells are what we call chondrocytes. chondrocytes were cultured in at $37^{\circ} \mathrm{C}$ and $5 \% \mathrm{CO}_{2}$. The study was reviewed and approved by the First Affiliated Hospital of Shenzhen University

117 Health Science Center Research Ethics Committee. All patients signed a written informed 118 consent before participating in this study.

\subsection{Toluidine blue staining of chondrocytes}


First, we washed the Petri dishes with PBS for 2 times and fixed the cells with 4\%

122 paraformaldehyde directly for about $0.5 \mathrm{~h}\left(4^{\circ} \mathrm{C}\right)$. Then wash with tap water for 15 minutes, then

123 with distilled water for 5 minutes, then add $1 \%$ toluidine blue for $2 \mathrm{~h}$. Finally, remove excess dye

124 and filter impurities. The dyeing condition was observed under the microscope, dried after

125 satisfaction, and sealed with neutral gum.

126

127

\subsection{Immunofluorescence staining of chondrocytes}

128 PBS washed the cells twice. The cells were fixed with paraformaldehyde for 15 min and 129 washed with PBS for 6 times / 5min. 0.5\% Triton-X100 was incubated and drilled for 15 min.

Wash PBS 6 times / 5min. The cells were sealed with 5\% BSA at room temperature for $2 \mathrm{~h}$ and cleaned with $0.5 \%$ BSA for 6 times / 5min. The primary antibody (Rabbit Anti-Collagen II/FITC Conjugated antibody (bs-10589R-FITC) and Rabbit Anti-Collagen I/Cy3 Conjugated antibody (bs-10423R-Cy3), Bioss, Beijing, China) were added and incubated at room temperature for $2 \mathrm{~h}$ and washed with PBS for 6 times / 5min. DAPI staining was added for 5min. Photos were taken using a confocal microscope ZEIZZ LSM800, Germany.

\subsection{Western Blot}

OA chondrocytes and normal control were cultured for three days, and then it was taken out of the $\mathrm{CO}_{2}$ incubator, cells were washed twice with PBS. Total protein was obtained by lysis buffer (Beyotime, China), and then placed on ice for 30 minutes. Centrifuged the sample at

$14112000 \mathrm{~g}$ for $25 \mathrm{~min}$ at $4^{\circ} \mathrm{C}$. The supernatants were transferred to a new EP tube. The protein

142 concentration was measured by BCA quantitative method. Protein samples were electrophoresed 143 on $10 \%$ SDS-PAGE gel. After that, protein samples were transferred on PVDF membranes 
144 according to the three-character sandwich method. Use 5\% skimmed milk powder to block for

145 2h. Use the primary antibody containing COL I (1:1000, boss, China), COL II (1:1000, Thermo

146 Scientific, USA) and GAPDH (1:1000, abcam, USA) to co-incubate with the sample overnight at

$1474{ }^{\circ} \mathrm{C}$. TBST cleaning, 5 times $/ 10 \mathrm{~min}$. Then use the second antibody containing goat anti-rabbit

148 incubation bath for two hours at room temperature. After washing the unbound antibody, use

149 chemiluminescence to take pictures in the two-color laser and chemiluminescence imaging 150 system (Odyssey FC, Gene Company Limited, China).

151

152 2.9 Apoptosis assay of chondrocytes

153 The chondrocytes were isolated from the cartilage tissue, washed and then resuspended, and

154 the precipitate was taken. About $50 \mathrm{uL}$ cell suspension was added into each flap-throwing tank 155 and centrifuged in a flap-throwing centrifuge (1500 g, $10 \mathrm{~min})$. The chondrocytes can be 156 attached to a slide after the surrounding water is absorbed by the filter paper. Then wash with 157 PBS for 3 times and fix with 4\% paraformaldehyde for 15min. Wash again with PBS for 3 times, 158 add PI immunofluorescence staining and incubate in a wet tank for $30 \mathrm{~min}$. After washing with 159 PBS again for 3 times, fluorescence microscope observation and image collection were 160 performed.

161

$162 \quad 2.10$ qRT-PCR Validation and Statistical Analysis

163 After previous research, we've found four hub genes, including MAPK14, PTPRC, B9D1, 164 PTPN12. We searched the primers of the four hub genes through the PrimerBank 165 (https://pga.mgh.harvard.edu/primerbank/) and ordered the relevant primers through the Sangon 166 Biotech. Quantitative real-time polymerase chain reaction primers are detailed in Table 1. Total 
167 RNA was extracted from chondrocytes using the RNeasy mini kit (Takara), according to the 168 manufacturer's instructions. The RNA was reversely transcribed into cDNA by iScriptTM cDNA 169 Synthesis Kit (Takara) and qRT-PCR was performed with a Real-time quantitative PCR 170 instrument (Life Technologies).

171

172

\subsection{Statistical analysis}

173 Use SigmaPlot 10.0 to draw. Use IBM SPSS Statistics 19 to conduct statistical analysis, data 174 are expressed as mean \pm std. A significant statistical difference was $\boldsymbol{P}<0.05$.

175

176

177

178

179

180

181

182

183

184

185

186

187

188

189

190

\section{Results}

\subsection{Identification of DEGs in Osteoarthritis}

A total of 23 OA knee joint samples and 22 matched normal knee joint samples were analyzed; taking $\boldsymbol{P}<0.05$ and $|\operatorname{logFC}|>1$ as a threshold, DEGs (2555 in GSE43923, 1347 in GSE55457 and 1398 in GSE11201) were identified. First, we made a pairwise comparison and got three Venn graphs (Figure 1A, B, C). Then we comprehensively compared and plotted the final Venn diagram of the differentially expressed gene (Figure 1 D). A total of 265 common differential genes were screened, including 66 down-regulated genes and 199 up-regulated genes.

\subsection{KEGG and GO enrichment analyses of DEGs}

By using DAVID, the DEGs functions were classified into 3 main categories, including BP, $\mathrm{MF}$, and $\mathrm{CC}$ (Figure 2). We can see that otherness in BP were significantly enriched in anatomical structure formation involved in morphogenesis, cell adhesion, vasculature development, blood vessel development (Figure 3A). Otherness in MF was mainly enriched in 
191 calcium channel inhibitor activity, signaling receptor binding, growth factor activity, cytoskeletal

192 protein binding and adenylate cyclase activator activity (Figure 3B). Otherness in CC was mainly

193 enriched in the cytoplasmic part, cytoplasm and extracellular region (Figure 3C).

194

195

\subsection{PPI network construction and module analysis}

We use the data obtained through STRING and then build the PPI network through 197 Cytoscape, containing 140 nodes and 356 edges (Figure 4). Next, we identified 4 hub genes, 198 including MAPK14, PTPN12, PTPRC and B9D1. Then we analyzed the 4 modules using MCODE (the plug-in for Cytoscape), the four modules were shown in (Figure 5). Functional enrichment analyses were also carried out for these modules. Pathway enrichment analysis showed that Module 1 is the signaling pathways regulating pluripotency of stem cells and mainly 202 associated with leukocyte transendothelial migration. Module 2 is mainly associated with the regulation of actin polymerization or depolymerization. Module 3 is mainly associated with the 204 positive regulation of response to external stimulus and leukocyte migration. It also can response 205 to lipopolysaccharide. Module 4 is mainly associated with the docking of the ciliary basal body206 plasma membrane (Table 2).

207

3.4 Morphological observation and authenticate of normal chondrocytes and OA 209 chondrocytes

210 When chondrocytes are completely adherent to the dish, they are mainly flat and polygonal. 211 The nuclei are located in the center of the cell, with 2-3 nucleoli. The morphology of OA 212 chondrocytes was more irregular, with rare cell community aggregation (Figure 6A). 
213 The cytoplasm of chondrocytes stained with toluidine blue was blue, and 1-3 purplish blue 214 nucleoli were observed (Figure 6B). The results showed that all the cells we cultured were 215 chondrocytes.

\subsection{Biological identification of $\mathrm{OA}$ and control chondrocytes}

218 COL I, COL II and DAPI immunohistochemical tested were carried out on the Chondrocytes. 219 And then we looked at it through a fluorescence microscope, the nuclei are dyed blue, collagen 220 type I dyed red, collagen type II dyed green. Results showed that OA chondrocytes compared 221 with normal chondrocytes, the COL I expression increased (Figure $6 \mathrm{C}-\mathrm{H}$ and $\mathrm{O}$ ), and the 222 expression of COL II reduced (Figure 6 I-N and O) detected by immunofluorescence and westernbolt. This indicates that OA chondrocytes mainly express collagen type I, which means that fibrochondrocytes are newly formed on the surface of cartilage, and the spontaneous depolymerization of fibrochondrocytes further accelerates the death of chondrocytes.

\subsection{Validation of Hub Gene}

4 hub genes have been found. Their details are shown in Table 3. We validated our results by 229 measuring the relative expression of our hub genes using qRT-PCR. As can be seen, the relative expression of MAPK-14, PTPN12 and PTPRC in OA chondrocytes were significantly increased, while the expression of B9D1 were significantly decreased $(\boldsymbol{P}<0.05)$ (Figure 7A-D), which was consistent with our expected results.

\subsection{Apoptosis test of chondrocytes}

The chondrocytes were stained with iodide propidium (PI) and examined by fluorescence 
237 chondrocytes were surrounded by pathological vesicles and showed intracellular red staining,

238 which was the PI entering the cells (Figure 7E-J). It suggested that the cell membrane was

239 incomplete and the cells death of OA chondrocytes was increased.

240

\section{Discussion}

242 The incidence of $\mathrm{OA}$ is a second after heart diseases in the statistical reports of some 243 countries. OA, cardiovascular, cerebrovascular diseases and cancer are also called "three major 244 killers" threatening human health. The most immediate symptom of OA is joint pain (Torres et 245 al. 2006). A large number of epidemiological studies have confirmed that osteoarthritis is a 246 complex disease involving multiple factors (Neogi \& Zhang 2013). Therefore, the molecular 247 mechanisms of OA are the most important direction we can study. It can help us to make early 248 diagnosis and treatment of OA.

249 In previous researches on bioinformatic analysis, many of them are only limited in two cohort 250 studies or two genetic events. This study makes up for the deficiency in this aspect, we used 3 251 datasets to obtain DEGs between OA knee joint samples and normal knee joint samples. This can 252 reduce the contingency of analysis. 265 DEGs were identified in all, which divided into 199 253 upregulated genes and 66 downregulated genes.

GO and KEGG enrichment analyses were performed found that the upregulated genes were mainly enriched in calcium channel inhibitor activity, cytoskeletal protein binding, adenylate cyclase activator activity, while the downregulated genes were mainly enriched in vasculature development, and blood vessel development. According to previous studies, calcium channels have been shown to mediate the OA pain pathway (Rahman et al. 2015). In addition, some studies have confirmed that the cytoskeletal structure is incomplete and diseased in cartilage chondrocytes of OA(Blain 2009). Other researches had indicated a protective role for pituitary 
261 adenylate cyclase-activating polypeptide (PACAP) in chondrocyte differentiation and 262 metabolism(Strange-Vognsen et al. 1997),(Juhasz et al. 2014),(Juhasz et al. 2015),(Juhasz et al. 263 2015). In addition, some studies have pointed out that one of the most important members of 264 osteogenesis is the skeletal vasculature and distinct blood vessel subtypes play different roles in 265 osteogenesis (Sivan et al. 2019),(Cui et al. 2016). Moreover, the enriched KEGG pathways of 266 DEGs and modules analysis included NF-kappa B signaling pathway, TNF signaling pathway, 267 PI3K-Akt signaling pathway and MAPK signaling pathway. Previous studies showed that PI3K268 Akt,NF-kappa B and TNF signaling pathways are involved in synovial hyperplasia and 269 osteoarthritis cartilage degeneration(Fu et al. 2016),(Lai et al. 2014) and changes in the 270 expression of cytokines, biological factors and enzymes on chondrocytes in human osteoarthritis 271 may be caused by the p38-MAPK signaling pathway(Feng et al. 2017). These theories are also 272 consistent with our results.

273 In this study, normal chondrocytes and OA chondrocytes were extracted from the collected 274 cartilage specimens of the knee joint of patients with femoral head and OA. The cytoplasm of 275 chondrocytes stained with toluidine blue was purplish blue, and 1-3 purplish blue nucleoli were 276 observed. we found that the expression levels of MAPK-14, PTPN12 and PTPRC in OA 277 chondrocytes were significantly increased, while the expression levels of B9D1 were 278 significantly decreased by qPCR test. This indicates that the changes in the expression level of 279 the potential key genes in the development of OA are consistent with the changes in the 280 expression level of OA chondrocytes, which is consistent with our conjecture.

281 However, does the high expression of MAPK-14 predict chondrocyte death? The chondrocyte 282 apoptosis experiment showed that the cell membrane of OA chondrocytes was more incomplete 283 than that of normal chondrocytes, and pathological vesicles appeared, indicating that the amount 
284 of necrosis of OA chondrocytes was significantly increased. It has been proved that chondrocytes

285 are doomed to die in the development of OA, while high expression of MAPK-14 plays a role in 286 inducing the autophagy of cells.

287 MAPK14 is one of the representatives of the MAPK family. Furthermore, the MAPK 288 signaling pathway is the most important signaling system to mediate osteoarthritic cartilage 289 damage (Chowdhury et al. 2008). Studies have shown that the major pathological changes of 290 osteoarthritis are degradation of the extracellular matrix, leading to progressive loss of cartilage 291 components and destruction of chondrocyte structure and function(Stoddart et al. 2009). 292 Inflammatory factors and growth factors specifically bind to receptors on cell membranes to 293 activate the intracellular MAPK signaling pathway, causing an increase in matrix 294 metalloproteinases (MMPs) expression, chondrocyte apoptosis and cartilage destruction. MMPs 295 can degrade almost all the extracellular matrix of chondrocytes and has been shown involved in 296 the pathogenesis of OA(Murphy et al. 2002). In addition, MAPK signaling pathway also 297 participates in and regulates chondrocyte enlargement, calcification and proliferation in the 298 process of OA pathology (Huang et al. 2008). Therefore, the MAPK signaling pathway can be 299 used as an effective target for the study of new drugs.

300 Other DEGs PRPRC and PTPN12 are members of the PTP family. On the basis of previous 301 studies, an important part of the pathogenesis of OA is the immune system(Goldring \& Otero 302 2011). The proliferation of $\mathrm{T}$ lymphocytes can regulate the immune response, and the 303 biochemical activities of human articular cartilage can directly affect the proliferation of $\mathrm{T}$ 304 lymphocytes(Pereira et al. 2016). T lymphocytes are lymphoid stem cells derived from bone 305 marrow and distributed to immune systems throughout the body through lymph and blood 306 circulation (Moro-Garcia et al. 2018). On the other hand, dendritic cells(DCs) as a crucial 
307 component in triggering an immune response, as well as in inflammatory and autoimmune 308 diseases(Ganguly et al. 2013). Furthermore, the PTP family is important in the activation of DCs 309 ability to induce T cell activation and function (Rhee et al. 2014). Therefore, the PTP family and 310 its related pathways may provide a new idea to treat OA.

311 The involvement of B9D1 in OA has not been well studied, but in recent research, mutations 312 of B9D1 result in common skeleton defects(Yuan \& Yang 2015). RB9D1 and its related 313 pathways can be used as a supplement to the current traditional treatment of OA.

314 In brief, four potential key genes in the pathogenesis of OA were identified through 315 bioinformatics screening, including MAPK-14, PTPRC, PTPN12 and B9D1. The imbalance of 316 matrix synthesis and catabolism of OA chondrocytes and the increase of cell membrane 317 destruction may be related to the high expression of MAPK-14 promoting the death of OA 318 chondrocytes. The regulation of the expression of these key genes can provide a potential 319 reference target for the treatment of OA.

320

321

\section{Conclusions}

322 The hub genes MAPK-14, PTPRC, PTPN12 were upregulated while B9D1 was 323 downregulated after screening from the 265 differentially expressed genes. OA chondrocytes had 324 high expression of COL I protein and low expression of COL II protein. The inflammatory 325 necrosis of OA chondrocytes was increased compared with the normal chondrocytes. The 326 expression levels of MAPK-14, PTPRC and PTPN12 in OA chondrocytes were significantly 327 higher, while the expression levels of B9D1 were significantly lower than that in normal 328 chondrocytes. The MAPK-14 among the four potential key genes has been proved to play an 329 important role in the treatment of OA. 
332 We thank Dr. Qian Yi, Dr. Weichao Sun, and Dr. Desheng Sun for their assistance in

333 bioinformatics and cell culture methods.

334

335

336 337

338

339

340

341

342

343

344

345

346

347

348

349

350

351

352

353

354

355

356

357

358

359

360

\section{1}

362

361

\section{Funding}

This study was supported by the National Natural Science Foundation of China (81800785, 81972085, 81772394), the Natural Science Foundation of Guangdong Province (2018A0303100027), the Sanming Project of Shenzhen Health and Family Planning Commission (SZSM201612086), Shenzhen Science and Technology Planning (JCYJ20180228163401333), and the Doctor Innovation Project of Shenzhen Health System (SZBC2018015), and Shenzhen Peacock Project (KQTD20170331100838136).

\section{Data Availability}

The following information was supplied regarding data availability:

Raw data are available in the Supplemental Files.

\section{Author Contributions}

Review conception and design: WL and DW.

Drafting of the article: ZD, XH and MA.

Drawing the schematic: $\mathrm{ZD}, \mathrm{XH}$, and MA.

Revising the manuscript: JL, MA, ZZ, XC, GW, JX, and DL.

Final approval of the article: All authors.

\section{Ethics approval and consent to participate}

The study was reviewed and approved by the First Affiliated Hospital of Shenzhen University Health Science Center Research Ethics Committee.

\section{Patient consent for publication}

All patients signed informed consent forms for publication.

\section{Competing interests}

The authors declare that they have no competing interests. 
363

364

365

366

367

368

369

370

371

372

373

374

375

376

377

378

379

380

381

382

383

384

385

386

387

388

389

390

391

392

393

\section{References}

Bader GD, and Hogue CW. 2003. An automated method for finding molecular complexes in large protein interaction networks. BMC Bioinformatics 4:2. 10.1186/1471-2105-4-2

Barrett T, Wilhite SE, Ledoux P, Evangelista C, Kim IF, Tomashevsky M, Marshall KA, Phillippy KH, Sherman PM, Holko M, Yefanov A, Lee H, Zhang N, Robertson CL, Serova N, Davis S, and Soboleva A. 2013. NCBI GEO: archive for functional genomics data sets--update. Nucleic Acids Res 41:D991-995. 10.1093/nar/gks1193

Blain EJ. 2009. Involvement of the cytoskeletal elements in articular cartilage homeostasis and pathology. Int J Exp Pathol 90:1-15. 10.1111/j.1365-2613.2008.00625.x

Chowdhury TT, Salter DM, Bader DL, and Lee DA. 2008. Signal transduction pathways involving p38 MAPK, JNK, NFkappaB and AP-1 influences the response of chondrocytes cultured in agarose constructs to IL-1beta and dynamic compression. Inflamm Res 57:306-313. 10.1007/s00011-007-7126-y

Cibrian Uhalte E, Wilkinson JM, Southam L, and Zeggini E. 2017. Pathways to understanding the genomic aetiology of osteoarthritis. Hum Mol Genet 26:R193-R201. $10.1093 / \mathrm{hmg} / \mathrm{ddx} 302$

Croft P. 2005. The epidemiology of osteoarthritis: Manchester and beyond. Rheumatology (Oxford) 44 Suppl 4:iv27-iv32. 10.1093/rheumatology/kei058

Cui Z, Crane J, Xie H, Jin X, Zhen G, Li C, Xie L, Wang L, Bian Q, Qiu T, Wan M, Xie M, Ding S, Yu B, and Cao X. 2016. Halofuginone attenuates osteoarthritis by inhibition of TGF-beta activity and H-type vessel formation in subchondral bone. Ann Rheum Dis 75:1714-1721. 10.1136/annrheumdis-2015-207923

Feng Z, Zheng W, Li X, Lin J, Xie C, Li H, Cheng L, Wu A, and Ni W. 2017. Cryptotanshinone protects against IL-1beta-induced inflammation in human osteoarthritis chondrocytes and ameliorates the progression of osteoarthritis in mice. Int Immunopharmacol 50:161-167. 10.1016/j.intimp.2017.06.017

Franceschini A, Szklarczyk D, Frankild S, Kuhn M, Simonovic M, Roth A, Lin J, Minguez P, Bork P, von Mering C, and Jensen LJ. 2013. STRING v9.1: protein-protein interaction networks, with increased coverage and integration. Nucleic Acids Res 41:D808-815. 10.1093/nar/gks1094 
394 Fu D, Shang X, Ni Z, and Shi G. 2016. Shikonin inhibits inflammation and chondrocyte

395

396

397

398

399

400

401

402

403

404

405

406

407

408

409

410

411

412

413

414

415

416

417

418

419

420

421

422

423

424 apoptosis by regulation of the PI3K/Akt signaling pathway in a rat model of osteoarthritis. Exp Ther Med 12:2735-2740. 10.3892/etm.2016.3642

Ganguly D, Haak S, Sisirak V, and Reizis B. 2013. The role of dendritic cells in autoimmunity. Nat Rev Immunol 13:566-577. 10.1038/nri3477

Goldring MB, and Otero M. 2011. Inflammation in osteoarthritis. Curr Opin Rheumatol 23:471478. 10.1097/BOR.0b013e328349c2b1

Hochberg MC, Yerges-Armstrong L, Yau M, and Mitchell BD. 2013. Genetic epidemiology of osteoarthritis: recent developments and future directions. Curr Opin Rheumatol 25:192197. 10.1097/BOR.0b013e32835cfb8e

Huang D, Ding Y, Luo WM, Bender S, Qian CN, Kort E, Zhang ZF, VandenBeldt K, Duesbery NS, Resau JH, and Teh BT. 2008. Inhibition of MAPK kinase signaling pathways suppressed renal cell carcinoma growth and angiogenesis in vivo. Cancer Res 68:81-88. 10.1158/0008-5472.CAN-07-5311

Huang DW, Sherman BT, Tan Q, Collins JR, Alvord WG, Roayaei J, Stephens R, Baseler MW, Lane HC, and Lempicki RA. 2007. The DAVID Gene Functional Classification Tool: a novel biological module-centric algorithm to functionally analyze large gene lists. Genome Biol 8:R183. 10.1186/gb-2007-8-9-r183

Juhasz T, Matta C, Katona E, Somogyi C, Takacs R, Gergely P, Csernoch L, Panyi G, Toth G, Reglodi D, Tamas A, and Zakany R. 2014. Pituitary adenylate cyclase activating polypeptide (PACAP) signalling exerts chondrogenesis promoting and protecting effects: implication of calcineurin as a downstream target. PLoS One 9:e91541. 10.1371/journal.pone.0091541

Juhasz T, Szentleleky E, Somogyi CS, Takacs R, Dobrosi N, Engler M, Tamas A, Reglodi D, and Zakany R. 2015. Pituitary Adenylate Cyclase Activating Polypeptide (PACAP) Pathway Is Induced by Mechanical Load and Reduces the Activity of Hedgehog Signaling in Chondrogenic Micromass Cell Cultures. Int J Mol Sci 16:17344-17367. 10.3390/ijms 160817344

Lai Y, Bai X, Zhao Y, Tian Q, Liu B, Lin EA, Chen Y, Lee B, Appleton CT, Beier F, Yu XP, and Liu CJ. 2014. ADAMTS-7 forms a positive feedback loop with TNF-alpha in the pathogenesis of osteoarthritis. Ann Rheum Dis 73:1575-1584. 10.1136/annrheumdis- 
425

426

427

428

429

430

431

432

433

434

435

436

437

438

439

440

441

442

443

444

445

446

447

448

449

450

451

452

453

454

455

2013-203561

McKenzie S, and Torkington A. 2010. Osteoarthritis - management options in general practice. Aust Fam Physician 39:622-625.

Moro-Garcia MA, Mayo JC, Sainz RM, and Alonso-Arias R. 2018. Influence of Inflammation in the Process of T Lymphocyte Differentiation: Proliferative, Metabolic, and Oxidative Changes. Front Immunol 9:339. 10.3389/fimmu.2018.00339

Murphy G, Knauper V, Atkinson S, Butler G, English W, Hutton M, Stracke J, and Clark I. 2002. Matrix metalloproteinases in arthritic disease. Arthritis Res 4 Suppl 3:S39-49. $10.1186 / \operatorname{ar} 572$

Neogi T, and Zhang Y. 2013. Epidemiology of osteoarthritis. Rheum Dis Clin North Am 39:1-19. 10.1016/j.rdc.2012.10.004

Pereira RC, Martinelli D, Cancedda R, Gentili C, and Poggi A. 2016. Human Articular Chondrocytes Regulate Immune Response by Affecting Directly T Cell Proliferation and Indirectly Inhibiting Monocyte Differentiation to Professional Antigen-Presenting Cells. Front Immunol 7:415. 10.3389/fimmu.2016.00415

Rahman W, Patel R, and Dickenson AH. 2015. Electrophysiological evidence for voltage-gated calcium channel 2 (Cav2) modulation of mechano- and thermosensitive spinal neuronal responses in a rat model of osteoarthritis. Neuroscience 305:76-85. 10.1016/j.neuroscience.2015.07.073

Rhee I, Zhong MC, Reizis B, Cheong C, and Veillette A. 2014. Control of dendritic cell migration, $\mathrm{T}$ cell-dependent immunity, and autoimmunity by protein tyrosine phosphatase PTPN12 expressed in dendritic cells. Mol Cell Biol 34:888-899. 10.1128/MCB.01369-13

Shannon P, Markiel A, Ozier O, Baliga NS, Wang JT, Ramage D, Amin N, Schwikowski B, and Ideker T. 2003. Cytoscape: a software environment for integrated models of biomolecular interaction networks. Genome Res 13:2498-2504. 10.1101/gr.1239303

Sivan U, De Angelis J, and Kusumbe AP. 2019. Role of angiocrine signals in bone development, homeostasis and disease. Open Biol 9:190144. 10.1098/rsob.190144

Song J, Chang RW, and Dunlop DD. 2006. Population impact of arthritis on disability in older adults. Arthritis Rheum 55:248-255. 10.1002/art.21842

Stoddart MJ, Grad S, Eglin D, and Alini M. 2009. Cells and biomaterials in cartilage tissue 
engineering. Regen Med 4:81-98. 10.2217/17460751.4.1.81

457 458

459

460

461

462

463

464

465

466

467

468

469

470
Strange-Vognsen HH, Arnbjerg J, and Hannibal J. 1997. Immunocytochemical demonstration of pituitary adenylate cyclase activating polypeptide (PACAP) in the porcine epiphyseal cartilage canals. Neuropeptides 31:137-141. 10.1016/s0143-4179(97)90082-2

Szklarczyk D, Franceschini A, Kuhn M, Simonovic M, Roth A, Minguez P, Doerks T, Stark M, Muller J, Bork P, Jensen LJ, and von Mering C. 2011. The STRING database in 2011: functional interaction networks of proteins, globally integrated and scored. Nucleic Acids Res 39:D561-568. 10.1093/nar/gkq973

Torres L, Dunlop DD, Peterfy C, Guermazi A, Prasad P, Hayes KW, Song J, Cahue S, Chang A, Marshall M, and Sharma L. 2006. The relationship between specific tissue lesions and pain severity in persons with knee osteoarthritis. Osteoarthritis Cartilage 14:1033-1040. 10.1016/j.joca.2006.03.015

Yuan X, and Yang S. 2015. Cilia/Ift protein and motor -related bone diseases and mouse models. Front Biosci (Landmark Ed) 20:515-555. 10.2741/4323 
Figure 1

Figure 1. Venn diagram.

A. GSE43923 and GSE55457 B. GSE11201 and GSE55457 C. GSE43923 and GSE11201 D. GSE43923, GSE55457 and GSE11201.
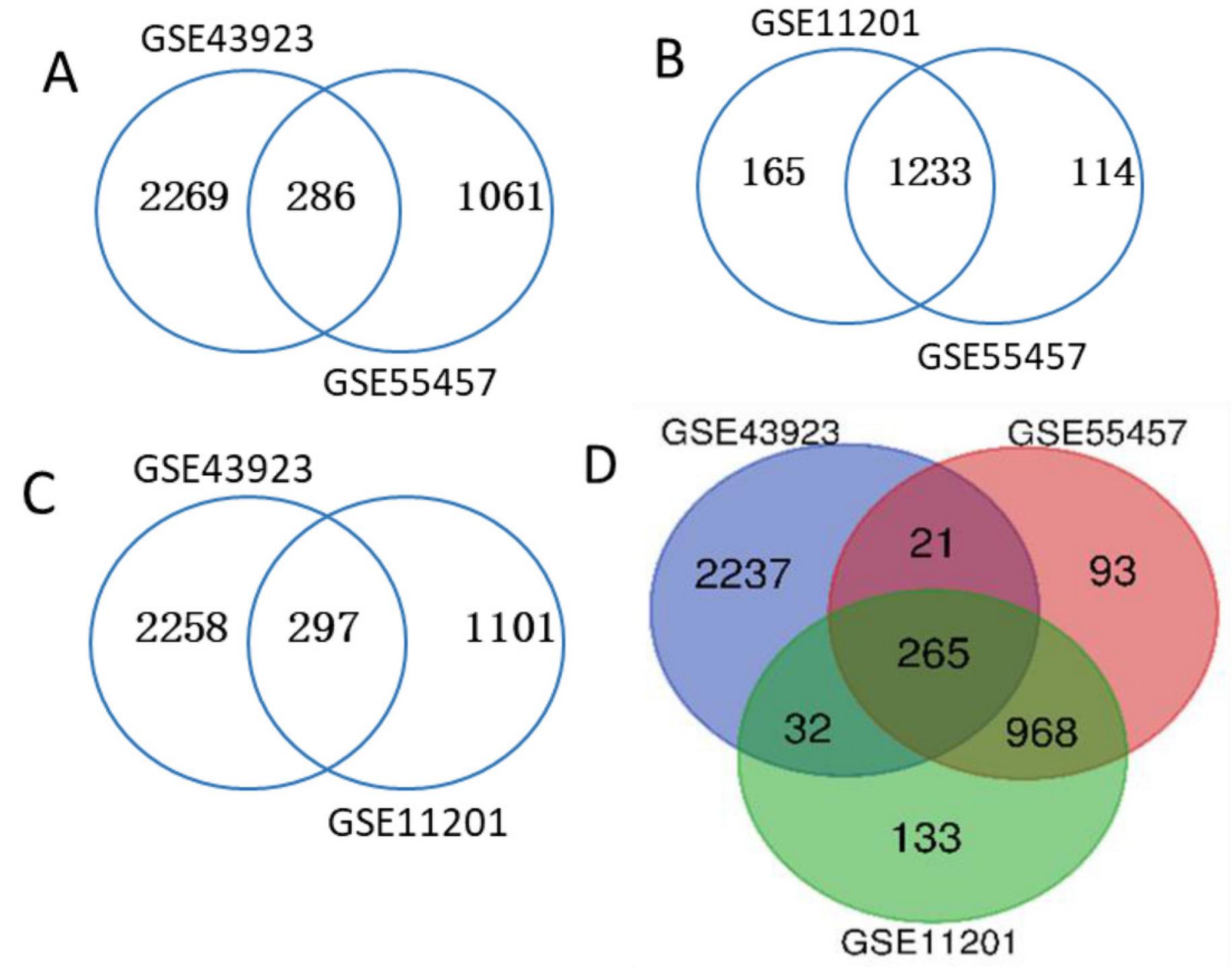
Figure 2

Figure 2. Gene Ontology (GO) analysis classified the DEGs into 3 groups: molecular function, biological process, and cellular component.

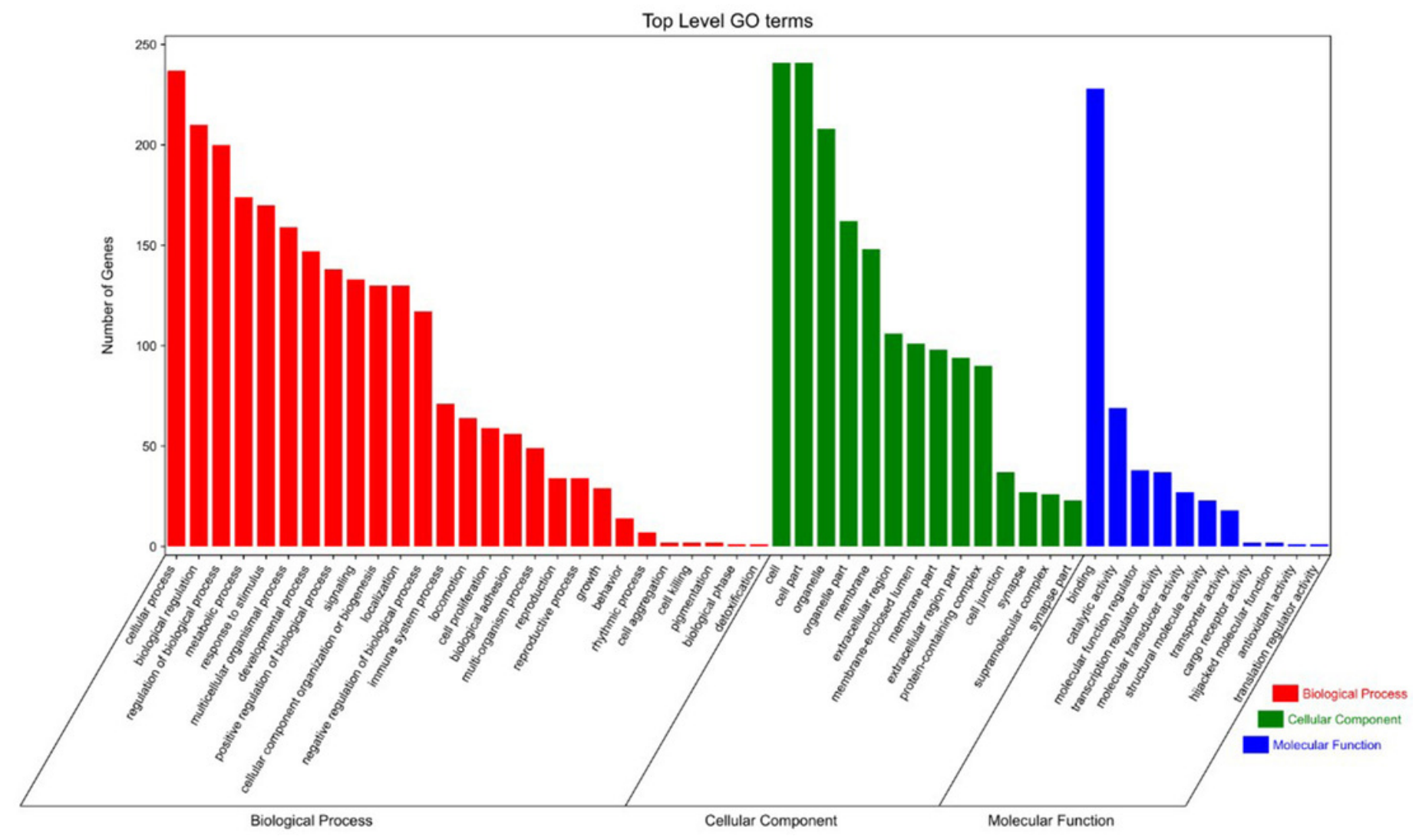




\section{Figure 3}

Figure 3. Gene Ontology (GO) enrichment analysis.

A Enrichment analysis of biological processes (BP). B Enrichment analysis of molecular function (MF). C Enrichment analysis of cellular component (CC). The gradual color represents the $\mathrm{P}$ value; the size of the black spots represents the gene number. 


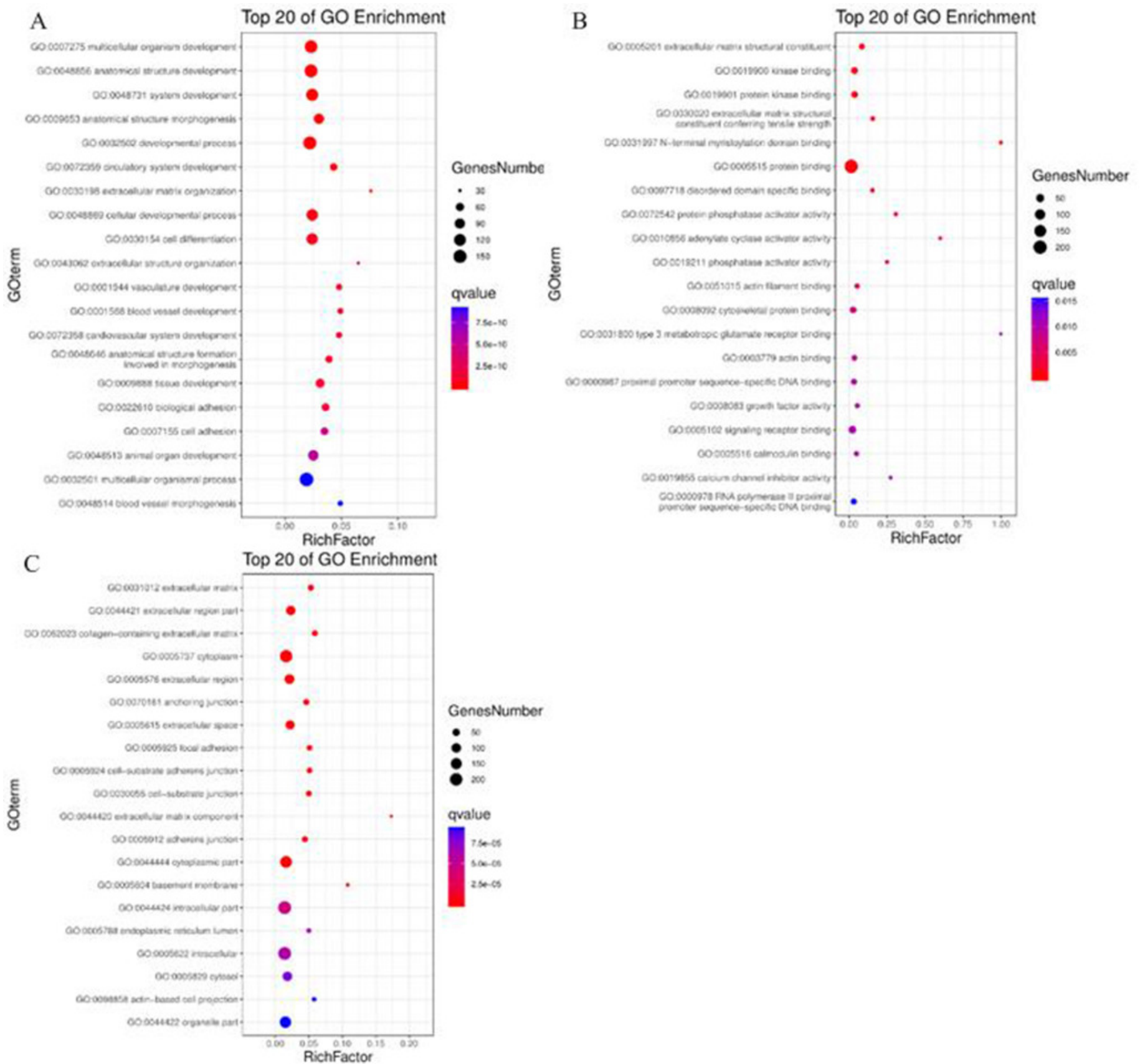


Figure 4

Fig 4. The PPI network of DEGs.

(Upregulated genes are marked in red; downregulated genes are marked in blue). 


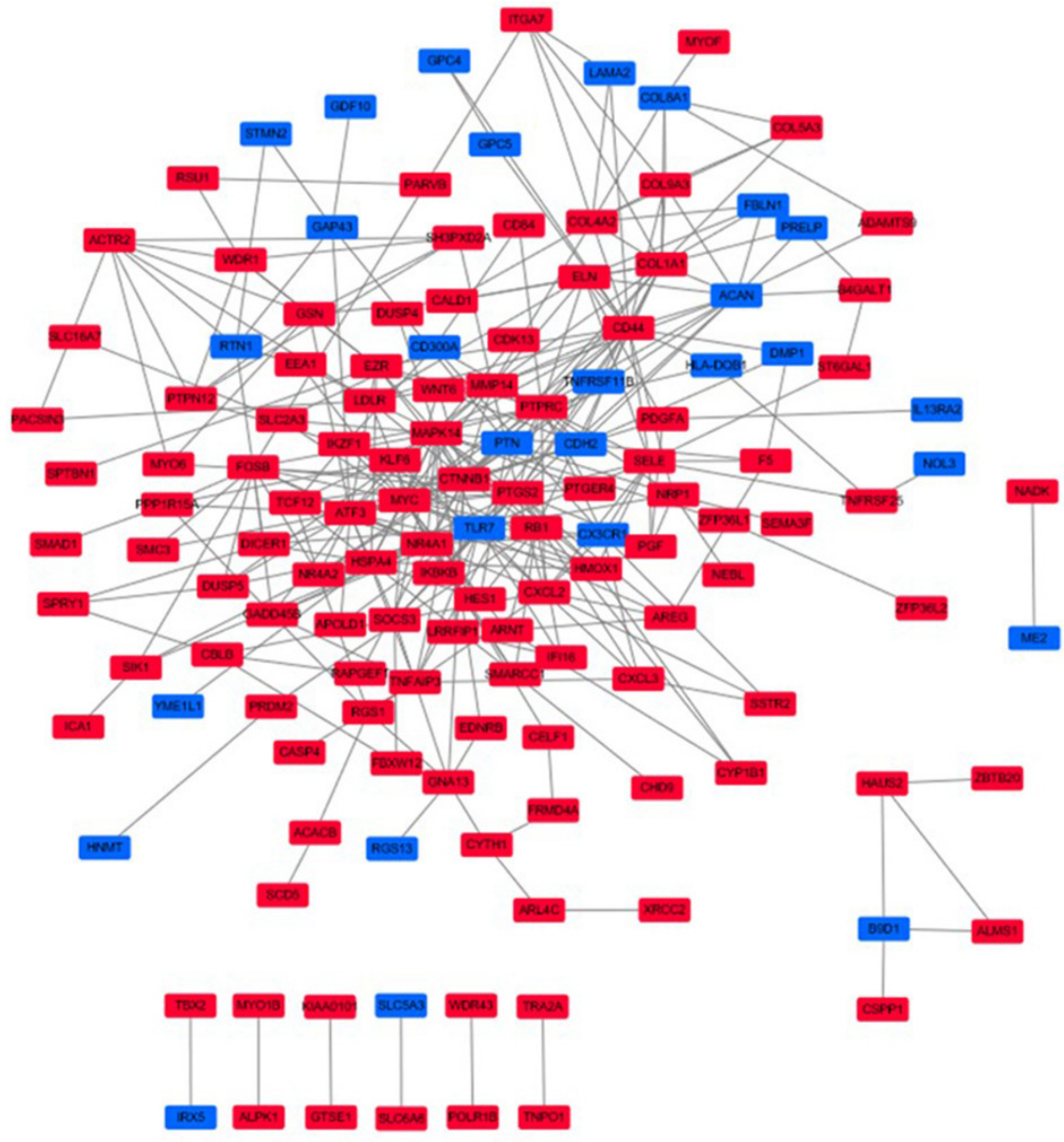




\section{Figure 5}

Figure 5. The 4 significant modules were obtained from PPI network.

Upregulated genes are marked in red; downregulated genes are marked in blue. A. MAPK14 module. B. PTPN12 module. C. PTPRC module. D. B9D1 module.

A

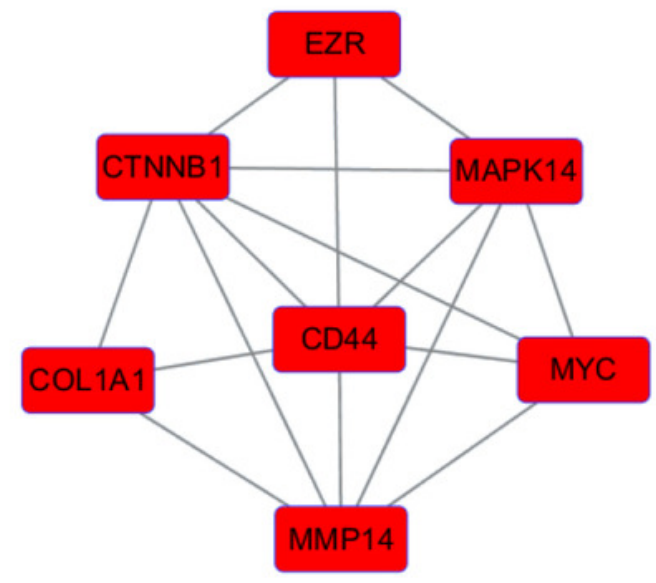

C

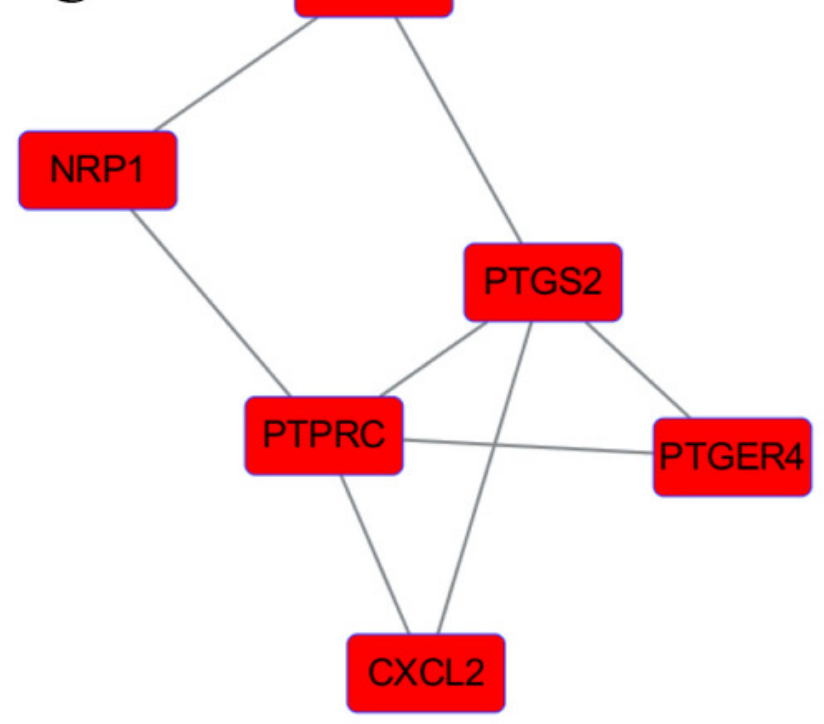

B

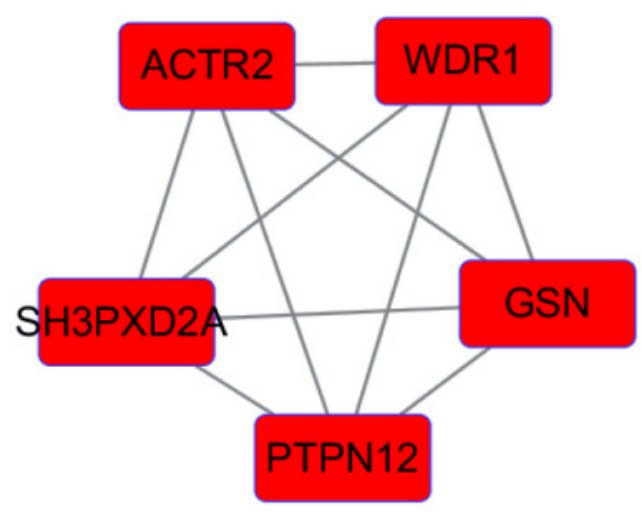

D

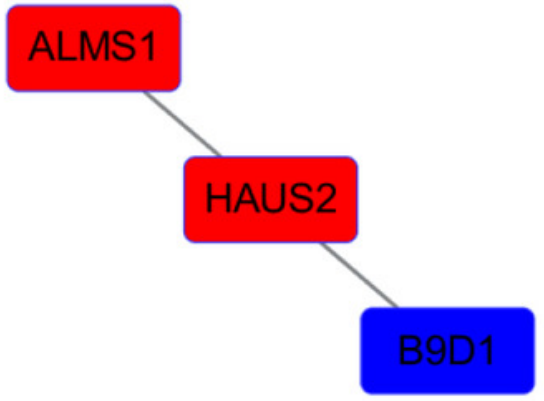




\section{Figure 6}

Figure 6. Cell biology detection.

A. Morphological observation of chondrocytes. B. Microscopic observation of toluidine blue staining of chondrocytes. C. - N. are the expression of COL I and COL II by immunofluorescence staining in human chondrocytes. $\mathbf{O}$. is the expression of $\mathrm{COL} I$ and $\mathrm{COL}$ II by fluorescence western blot in human chondrocytes. 


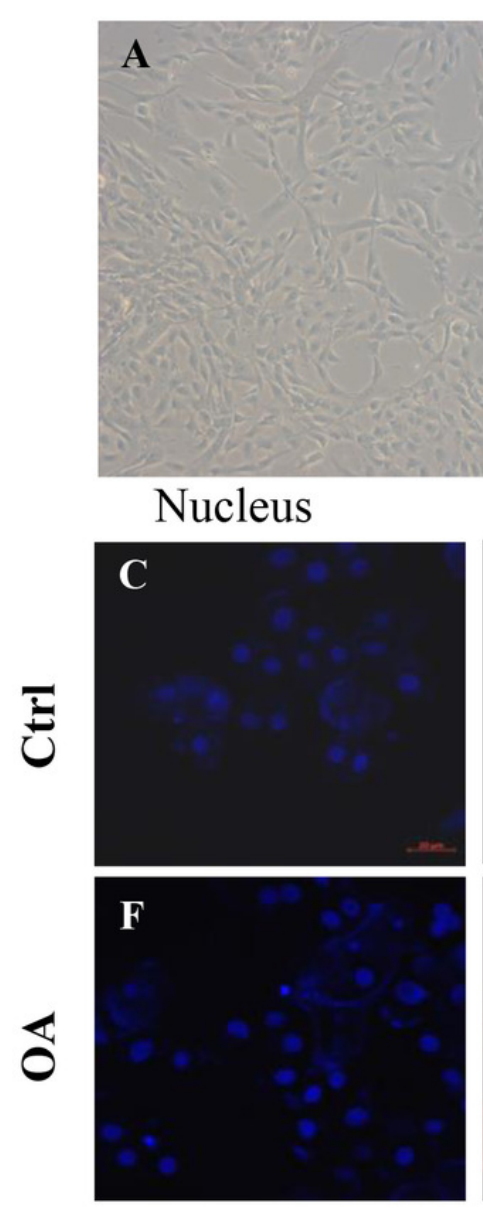

Nucleus
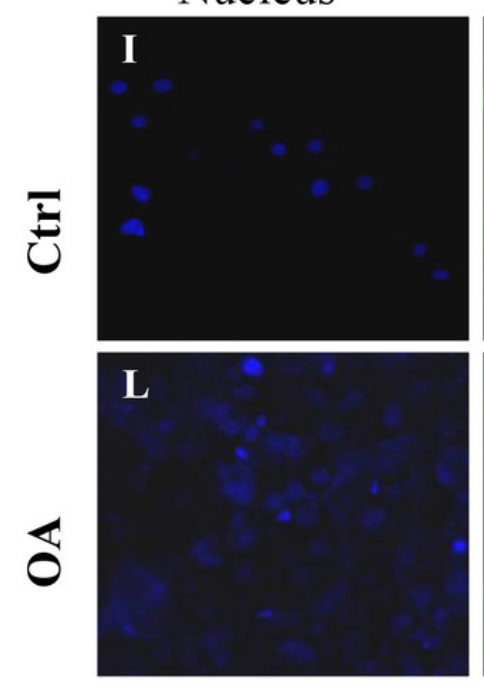

O

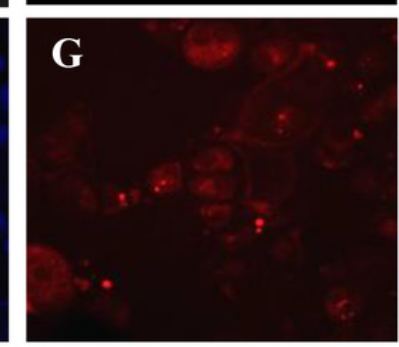

COL II

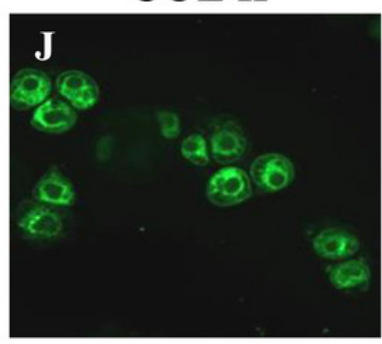

M
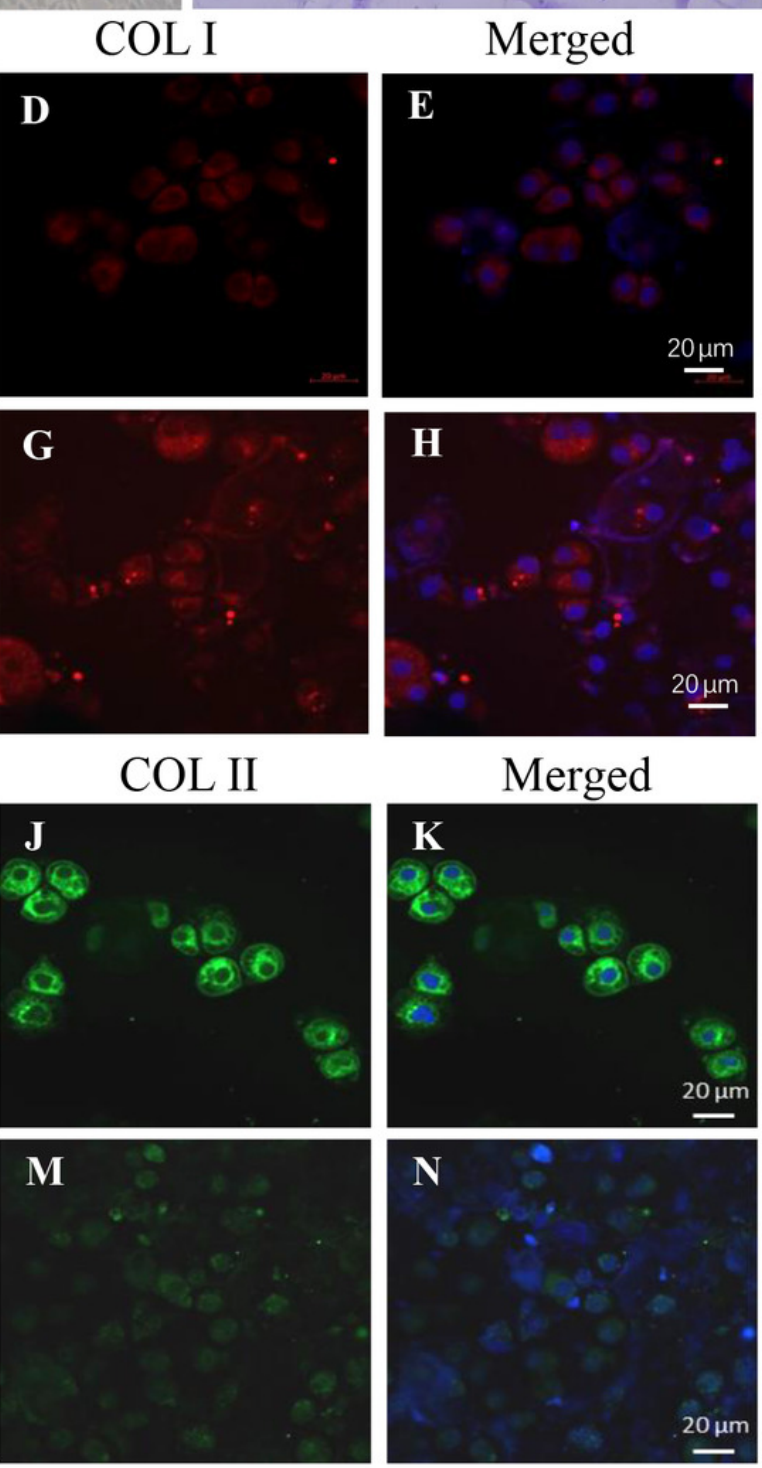

N

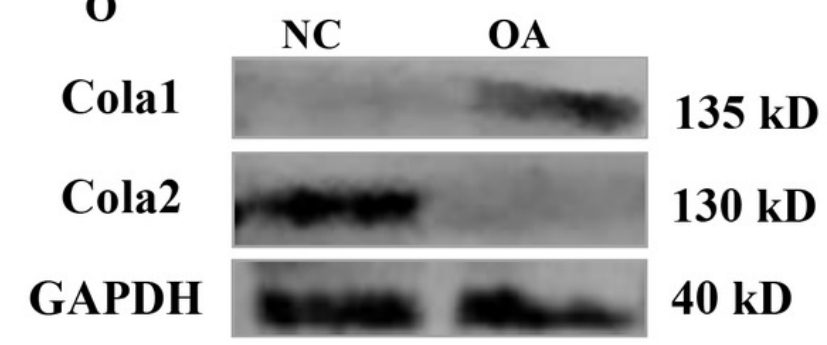

Peer] reviewing PDF | (2020:06:49914:1:2:NEW 20 Nov 2020) 


\section{Figure 7}

Figure 7. Comparison of expression of potential key genes and Apoptosis test in chondrocytes.

(A. MAPK-14; B. PTPRC; C. PTPN12; D. B9D1). All samples were normalized to the expression of GAPDH, and the relative expression levels of each gene were analyzed using the $2^{\wedge} \Delta \Delta C T$ method. E.-J. Apoptosis test of chondrocytes (DIC: bright field imaging; PI: piridine iodide staining imaging; Merged: composite imaging). The red arrow refers to cells that are stained or not by PI. 
A

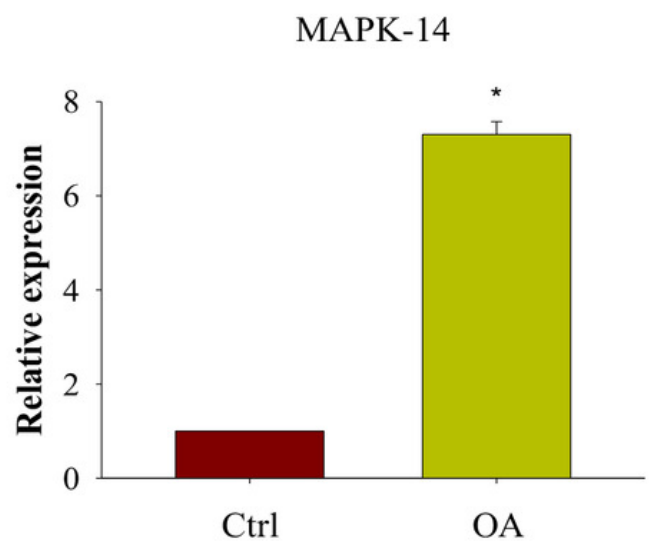

C

PTPRC
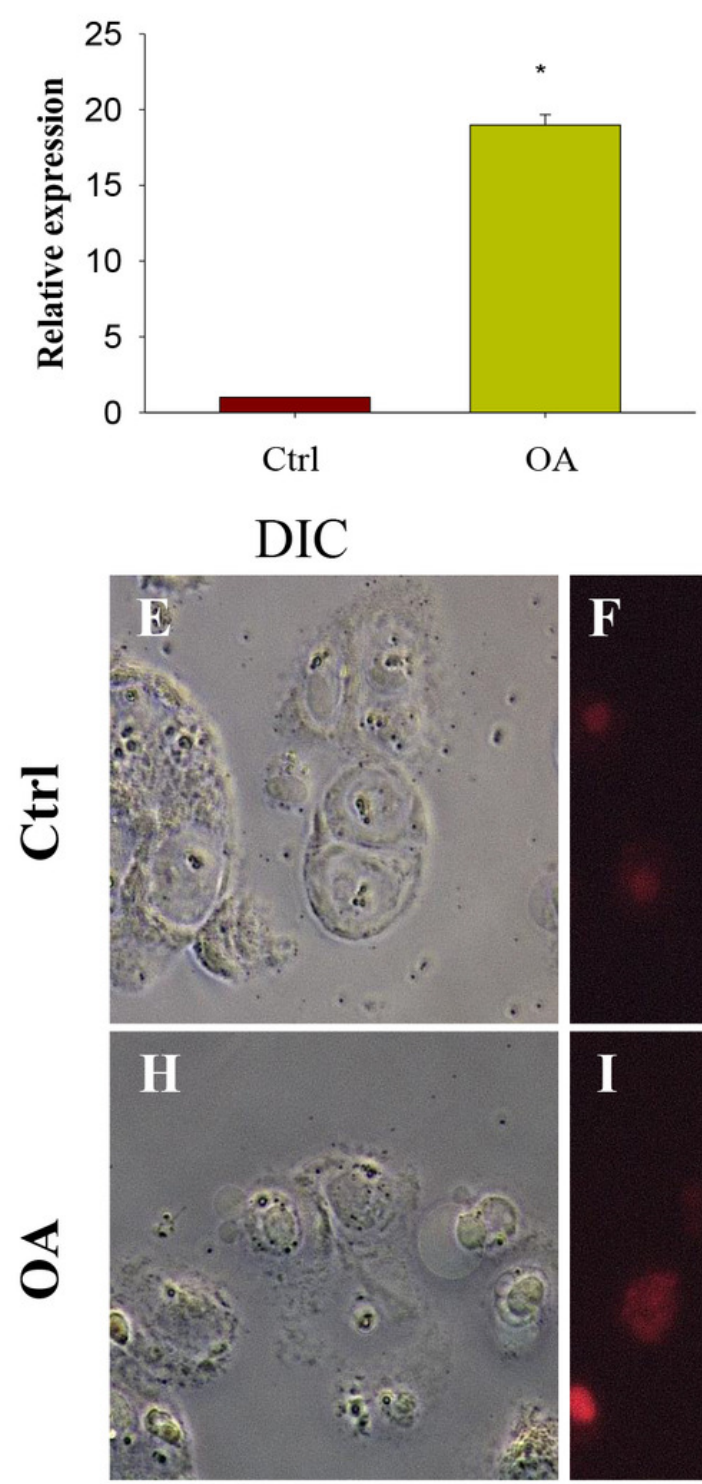

B
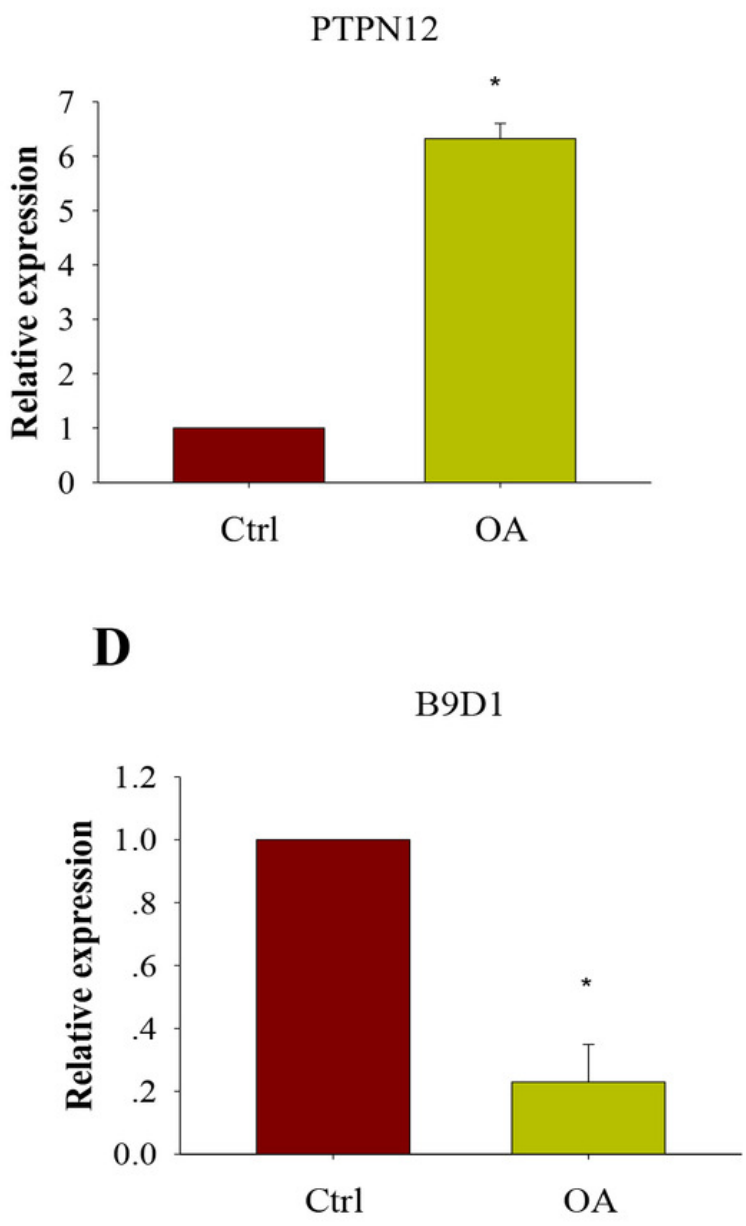

PI
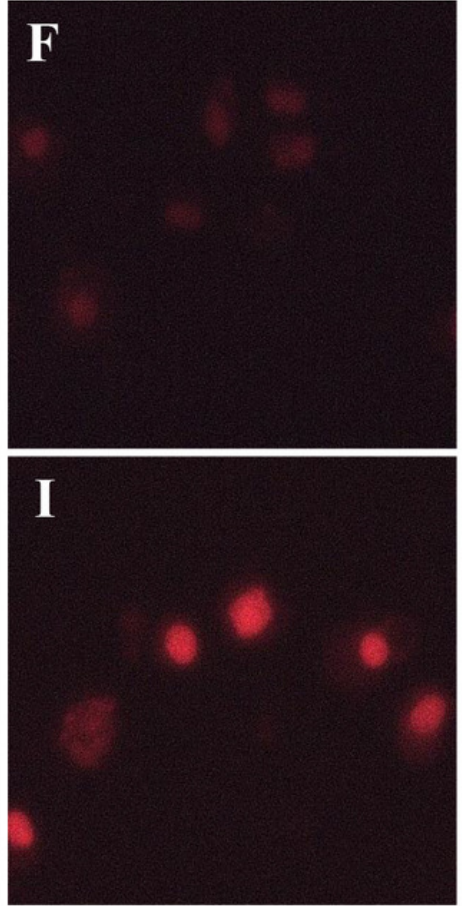

Merged
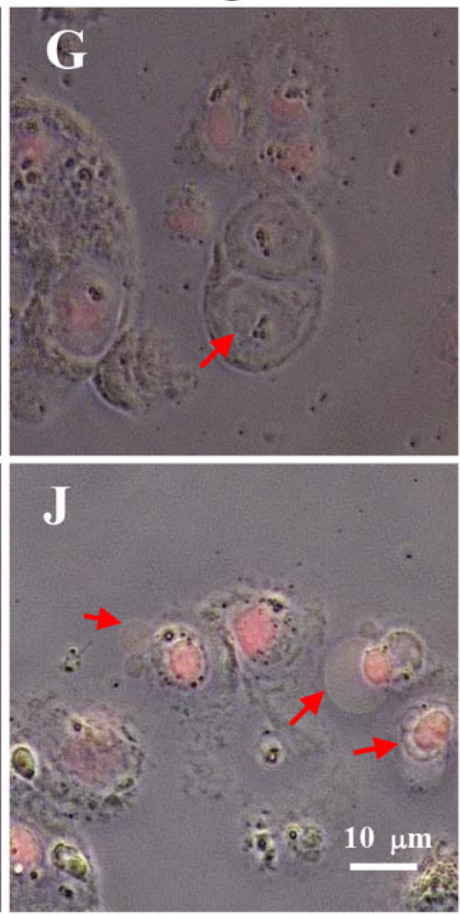


\section{Table 1 (on next page)}

Table 1: The primers of 4 hub genes 
1

Table 1: The primers of 4 hub genes

\begin{tabular}{|c|c|c|c|c|}
\hline Gene & Primer sequence & $\begin{array}{l}\text { Annealing } \\
\text { Temperat } \\
\left({ }^{\circ} \mathrm{C}\right) \\
\end{array}$ & $\begin{array}{l}\text { Length } \\
\text { e }\end{array}$ & $\begin{array}{c}\text { PrimerBank } \\
\text { ID }\end{array}$ \\
\hline \multirow{2}{*}{ MAPK14 } & Fwd 5'- CCCGAGCGTTACCAGAACC -3' & 62.4 & 19 & \multirow[t]{2}{*}{$194578904 \mathrm{cl}$} \\
\hline & Rev 5'- TCGCATGAATGATGGACTGAAAT-3' & 60.4 & 23 & \\
\hline \multirow{2}{*}{ PTPRC } & Fwd 5'- ACCACAAGTTTACTAACGCAAGT-3' & 60.4 & 23 & \multirow[t]{2}{*}{$18641362 \mathrm{a} 1$} \\
\hline & Rev 5'- TTTGAGGGGGATTCCAGGTAAT-3 & 60.5 & 22 & \\
\hline \multirow{2}{*}{ B9D1 } & Fwd 5'- TGGAGGAGGGGATCTCACAG-3' & 61.9 & 20 & \multirow[t]{2}{*}{$343478278 \mathrm{c} 1$} \\
\hline & Rev 5'- CCGTAGGGGTTGGTGCTTTT-3 & 62.4 & 20 & \\
\hline \multirow{2}{*}{ PTPN12 } & Fwd 5'- AGTTGCCTTGTTGAAGGGGAT-3' & 61.6 & 21 & $196114950 \mathrm{c} 1$ \\
\hline & Rev 5' - AGAAGGTGTCAAGATGGGTGG-3 & 61.4 & 21 & $\square$ \\
\hline
\end{tabular}

2 


\section{Table 2 (on next page)}

Table 2 Signaling pathway enrichment analysis of hub DEGs function in OA 
1

Table 2 Signaling pathway enrichment analysis of hub DEGs function in OA

\begin{tabular}{|c|c|c|c|c|}
\hline Expression & Term & Description & $\begin{array}{l}\text { Gene } \\
\text { Count }\end{array}$ & $\log P$ \\
\hline \multirow[t]{5}{*}{ UP-DEGs } & hsa04550 & $\begin{array}{c}\text { Signaling pathways regulating pluripotency of } \\
\text { stem cells }\end{array}$ & 3 & -5.43 \\
\hline & hsa04670 & Leukocyte transendothelial migration & 3 & -5.69 \\
\hline & GO:0008064 & $\begin{array}{c}\text { regulation of actin polymerization or } \\
\text { depolymerization }\end{array}$ & 3 & -5.4 \\
\hline & GO:0032103 & $\begin{array}{l}\text { positive regulation of response to external } \\
\text { stimulus }\end{array}$ & 3 & -6.33 \\
\hline & GO:0097529 & myeloid leukocyte migration & 3 & -4.88 \\
\hline$\square$ & GO:0032496 & response to lipopolysaccharide & 3 & -4.31 \\
\hline $\begin{array}{l}\text { DOWN- } \\
\text { DEGs }\end{array}$ & GO:0097711 & ciliary basal body-plasma membrane docking & 3 & -7.23 \\
\hline
\end{tabular}

2

3

4 
Table 3 (on next page)

Table 3. Functional roles of 4 hub genes 
1

Table 3. Functional roles of 4 hub genes

\begin{tabular}{|c|c|c|c|}
\hline No. & $\begin{array}{c}\text { Gene } \\
\text { symbol }\end{array}$ & Full name & Function \\
\hline 1 & $\begin{array}{l}\text { MAPK- } \\
14\end{array}$ & $\begin{array}{l}\text { mitogen-activated } \\
\text { protein kinase } 14\end{array}$ & $\begin{array}{l}\text { Play an important role in the cascades of } \\
\text { cellular responses evoked and inhibit the } \\
\text { lysosomal degradation pathway of autophagy }\end{array}$ \\
\hline 2 & PTPRC & $\begin{array}{l}\text { protein tyrosine } \\
\text { phosphatase receptor } \\
\text { type } \mathrm{C}\end{array}$ & $\begin{array}{l}\text { High expression of PTPRC is associated with } \\
\text { progression of PTP family }\end{array}$ \\
\hline 3 & PTPN12 & $\begin{array}{l}\text { protein tyrosine } \\
\text { phosphatase non- } \\
\text { receptor type } 12\end{array}$ & $\begin{array}{l}\text { High expression of PTPN12 is associated } \\
\text { with progression of PTP family }\end{array}$ \\
\hline 4 & B9D1 & $\begin{array}{l}\text { B9 domain } \\
\text { containing } 1\end{array}$ & $\begin{array}{l}\text { Component of the tectonic-like complex and } \\
\text { acting as a barrier that prevents diffusion of } \\
\text { transmembrane proteins between the cilia and } \\
\text { plasma membranes }\end{array}$ \\
\hline
\end{tabular}

